ORIGINAL ARTICLE

\title{
Lack of vascular adventitial fibroblastic cells in tumour stroma of intestinal-type and solid-type gastric carcinomas
}

\author{
H Nakayama, H Enzan, E Miyazaki, N Kuroda, M Toi, M Hiroi, W Yasui
}

See end of article for

authors' affiliations

J Clin Pathol 2004;57:183-185

Correspondence to: Dr H Nakayama, Department of Molecular

Pathology, Graduate

School of Biomedical

Sciences, Hiroshima

University, 1-2-3, Kasumi,

Minami-ku, Hiroshima

734-8551, Japan;

nakayamh@

hiroshima-u.ac.jp

Accepted for publication 13 August 2003

\begin{abstract}
Aims: To investigate the roles of vascular adventitial fibroblastic cells in tumour stroma, the distribution of vascular adventitial fibroblastic cells was studied in gastric carcinomas.

Methods: In total, 50 surgically resected gastric carcinomas (43 intestinal type, and seven solid type) and their normal tissues were examined. Vascular adventitial fibroblastic cells are positive for CD34 but negative for CD31. To differentiate vascular adventitial fibroblastic cells from vascular endothelial cells, immunostaining for CD34 and CD31 was performed. Immunostaining for high molecular weight caldesmon was also performed to recognise vascular media.

Results: In normal gastric tissues, CD34 positive fibroblastic cells were found just outside the vascular media, namely vascular adventitial fibroblastic cells. In contrast, all of the 43 intestinal-type and seven solid-type gastric carcinomas had no vascular adventitial fibroblastic cells in the tumour stroma.

Conclusions: These results suggest that a lack of vascular adventitial fibroblastic cells is associated with fumour stroma formation in intestinal-type and solid-type gastric carcinomas.
\end{abstract}

$\mathrm{T}$ he vascular adventitia, located just outside the vascular media, is a poorly defined layer of connective tissue in which nerve fibres, some smooth muscle cells, fibroblastic cells, and the vasa vasorum are found. ${ }^{1}$ Vascular adventitial fibroblastic cells are positive for CD $34,{ }^{23}$ which is a $110 \mathrm{kDa}$ transmembrane cell surface glycoprotein, ${ }^{4}$ and was originally described as a marker for human haemopoietic stem cells. ${ }^{5}$

"To date, the vascular adventitia of tumour stroma has not been studied extensively"

The vascular adventitia of tumour vessels is a very important component, from the point of view of angiogenesis and vessel wall stabilisation. However, to date, the vascular adventitia of tumour stroma has not been studied extensively.

Therefore, to elucidate the roles of vascular adventitial fibroblastic cells in the tumour stroma of gastric carcinomas, we examined the distribution of vascular adventitial fibroblastic cells in gastric carcinomas and their normal tissues using CD34 as an immunohistochemical marker for vascular adventitial fibroblastic cells.

\section{MATERIALS AND METHODS}

We examined 50 surgically resected invasive gastric carcinomas, which were confined to the gastric wall (not invading the adjacent organs), and their normal tissues from the surgical pathology files of the first department of pathology, Kochi Medical School, Japan and its affiliated hospitals from 1994 to 2001. The definitions used for histological classification were based on the criteria of Lauren ${ }^{6}$ and our previous report. ${ }^{7}$ We studied 43 intestinal-type tumours and seven solid-type tumours. We excluded diffuse-type carcinomas because these tumours spread in the muscle coat and subserosa without an increase in stromal components $^{6}$ and involve pre-existing normal vessels, making it very difficult to discriminate tumour vessels from normal vessels.
The maximum tumour cut surface was immunostained in all 50 tumours; we used one to four paraffin wax blocks for each case.

Immunohistochemical studies were performed by the streptavidin biotin method using the Histofine SAB-PO $(\mathrm{M})$ kit (Nichirei, Tokyo, Japan). Three monoclonal antibodies against CD34 (clone MY10; 1/20 dilution; no pretreatment; Becton-Dickinson, Lexington, North Carolina, USA), CD31 (clone JC/70A; 1/20 dilution; pronase pretreatment; Dakopatts, Glostrup, Denmark), and high molecular weight caldesmon (HCD; clone h-CD; 1/50 dilution; microwave pretreatment; Dakopatts) were used. We also examined immunoreactivity for CD31 in all of the tumours and their normal tissues, to distinguish CD34 positive vascular adventitial fibroblastic cells from vascular endothelial cells, which are positive for both CD34 and CD31.8 Vascular endothelial cells were used as the internal positive control of immunostaining for CD34 and CD $31 .{ }^{8}$ Immunostaining for $\mathrm{HCD}$ was also performed, to recognise the vascular media in each specimen examined.

We assessed the areas just outside the vascular media in the whole maximum tumour cut surface, except the tumour growing edge. The tumours were classified into two groups, namely: (-), tumours with no CD34 positive fibroblastic cells just outside the vascular media; and $(+)$, tumours with more than one CD34 positive fibroblastic cell just outside the vascular media. Two independent attending pathologists (HN and HE) examined all the immunostained specimens randomly. After evaluation by the two independent pathologists, all seven authors discussed the results to make them as objective as possible.

\section{RESULTS}

Table 1 summarises the results.

CD34 positive vascular adventitial fibroblastic cells surrounded the vascular media in normal gastric tissue; muscle

Abbreviations: HCD, high molecular weight caldesmon; $\alpha-S M A$, $\alpha$ smooth muscle actin 


\begin{tabular}{|c|c|c|c|}
\hline \multirow[b]{2}{*}{ Tumour } & \multirow[b]{2}{*}{ Number of cases } & \multicolumn{2}{|c|}{ VAFC } \\
\hline & & $(-1$ & $1+1$ \\
\hline \multicolumn{4}{|l|}{ Histology } \\
\hline Intestinal type & 43 & 43 & 0 \\
\hline Solid type & 7 & 7 & 0 \\
\hline
\end{tabular}

$(-)$, absence of vascular adventitial fibroblastic cells; $(+)$, presence of vascular adventitial fibroblastic cells.

arteries, small arteries, arterioles, venules, and veins had CD34 positive vascular adventitial fibroblastic cells (fig l; note: figs 2 and 3 show the expression of CD31 and high molecular weight caldesmon, respectively, at the same site as fig 1).

In all of the 43 intestinal-type and seven solid-type carcinomas examined, no CD34 positive fibroblastic cells were detected just outside the vascular media. No vascular adventitial fibroblastic cells were detected. CD34 was positive only in vascular endothelial cells (fig 4; note: fig 5 shows the expression of CD31 at the same site as fig 4). As reported previously, ${ }^{9}$ the desmoplastic stromal cells in the 50 gastric carcinomas studied here were all negative for CD34.

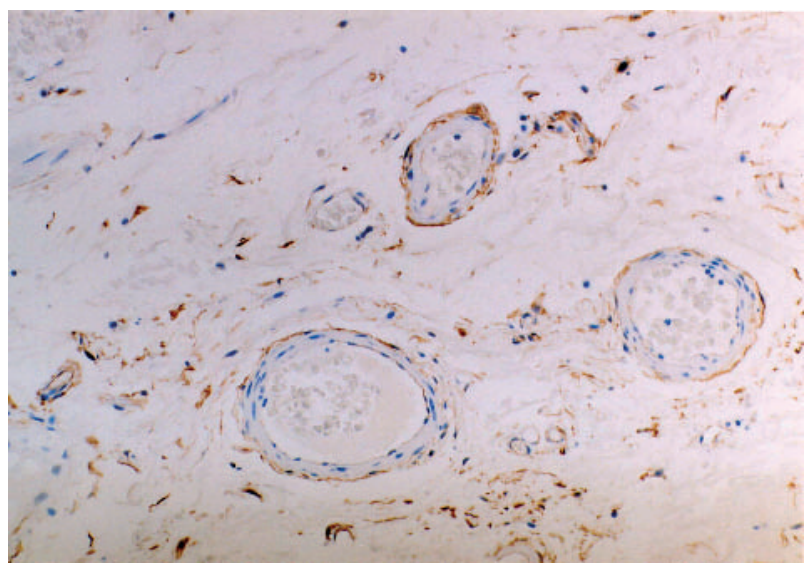

Figure 1 The presence of vascular adventitial fibroblastic cells in normal gastric tissue. CD34 positive fibroblastic cells (vascular adventitial fibroblastic cells) surround the vascular media (see fig 3).

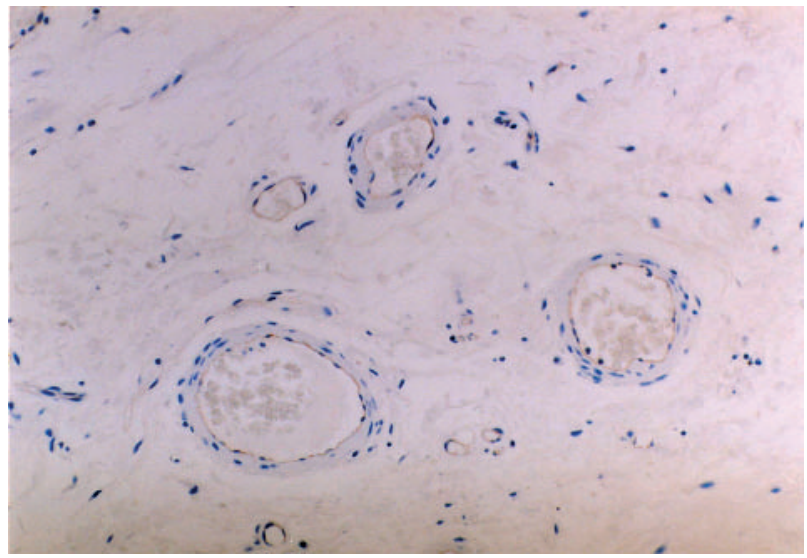

Figure 2 Normal gastric tissue: staining for CD31 to distinguish CD34 positive stromal cells from vascular endothelial cells.

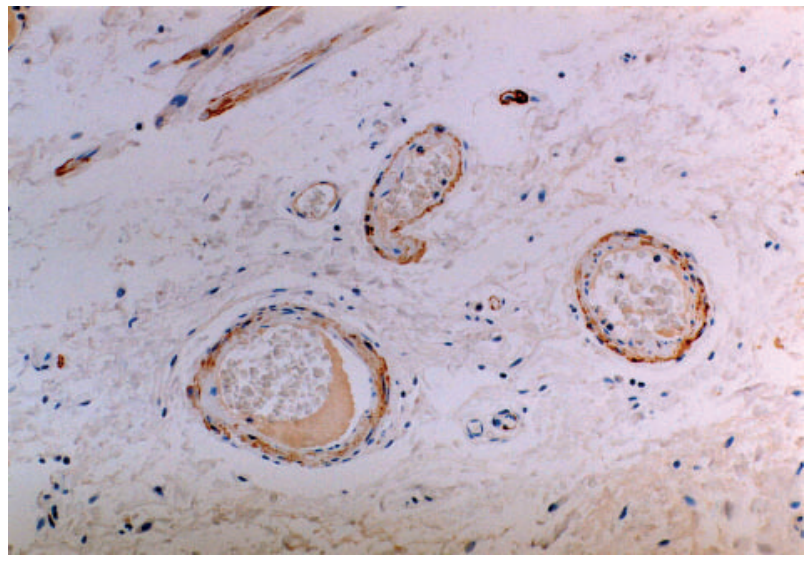

Figure 3 Normal gastric tissue: staining for high molecular weight caldesmon to highlight the vascular media.

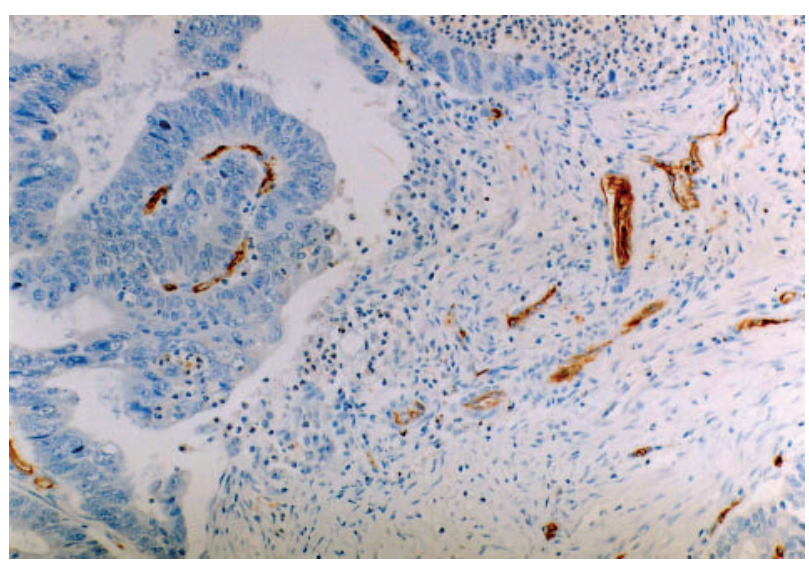

Figure 4 Staining for CD34 in intestinal-type gastric carcinoma showed the lack of CD34 positive fibroblastic cells (vascular adventitial fibroblastic cells) just outside the vascular media (see fig 5).

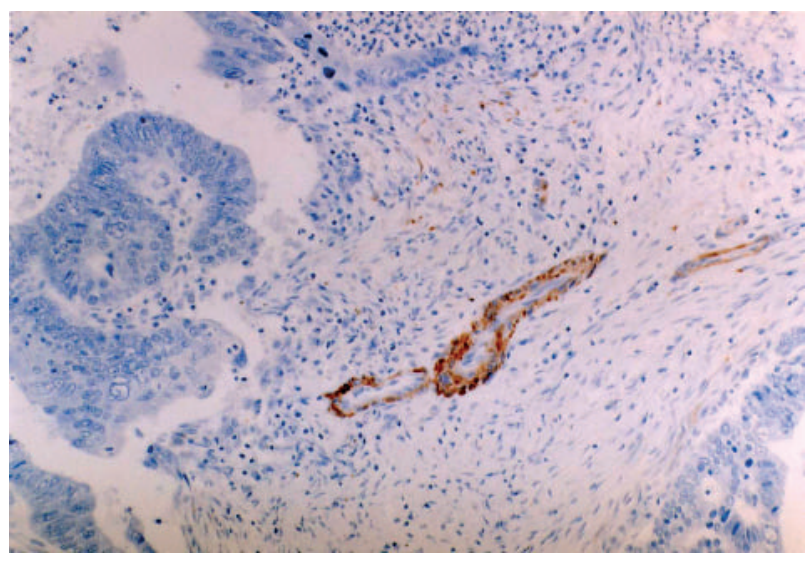

Figure 5 Staining for high molecular weight caldesmon in intestinal-type gastric carcinoma to highlight the vascular media.

\section{DISCUSSION}

The vessel wall is composed of three layers, namely: intima, media, and adventitia. The adventitial layer has always been thought of as a supporting tissue of vessels. 


\section{Take home messages}

- CD34 positive vascular adventitial fibroblastic cells were present just outside the vascular media in normal gastric tissues

- No vascular adventitial fibroblastic cells were found outside the stroma in the 43 intestinal-type and seven solid-type gastric carcinomas studied

- A lack of vascular adventitial fibroblastic cells may be associated with tumour stroma formation in intestinaltype and solid-type gastric carcinomas

- Further investigations are required to substantiate this hypothesis

Recently, however, the role of the adventitia in the context of providing cells and molecules with the capacity to influence neointima formation and vascular remodelling has received considerable attention in cardiovascular disorders. ${ }^{10}$ In our present study, we focused on vascular adventitial fibroblastic cells in tumour stroma. To our knowledge, this is the first report regarding vascular adventitial fibroblastic cells in the tumour stroma of malignant epithelial tumours.

Tumour stromal cells in intestinal-type and solid-type advanced gastric carcinomas are all negative for CD34, whereas they are positive for $\alpha$ smooth muscle actin $\left(\alpha\right.$-SMA). ${ }^{79}$ In our present study, we found no vascular adventitial fibroblastic cells in the tumour stroma of intestinal-type and solid-type gastric carcinomas. Intestinaltype and solid-type gastric carcinomas have desmoplastic stromal cells, which are positive for $\alpha$-SMA within tumour tissue. It is possible that the lack of vascular adventitial cells is associated with a desmoplastic stromal reaction (the presence of $\alpha$-SMA positive stromal cells). ${ }^{7}$

Vascular adventitia is a poorly defined layer of connective tissue. Vascular peri-adventitia is also an ill defined sheath of loose connective tissue just outside the vascular adventitia ${ }^{1}$ Vascular peri-adventitial fibroblastic cells are also positive for CD $34 .{ }^{2}{ }^{3}$ In our study, no CD34 positive fibroblastic cells were detected in the vascular wall or around the vessels. The lack of vascular adventitial fibroblastic cells in the tumour stroma of intestinal-type and solid-type gastric carcinomas was objectively confirmed in our present immunohistochemical study.

In conclusion, the tumour stromal vessels of the intestinaltype and solid-type gastric carcinomas examined contained no adventitial fibroblastic cells. There is a possibility that the lack of vascular adventitial fibroblastic cells is associated with tumour stroma formation in intestinal-type and solid-type gastric carcinomas. To elucidate the pathobiological relevance of vascular adventitial fibroblastic cells in normal stromal tissue and tumour stroma formation, further molecular and biological investigations are needed.

\section{ACKNOWLEDGEMENTS}

The authors are grateful to Ms H Yamasaki, Ms M Yamamoto, Mr T Tokaji, and Mr Y Hayashi, First Department of Pathology, Kochi Medical School, for their excellent technical assistance.

\section{Authors' affiliations}

H Nakayama, W Yasui, Department of Molecular Pathology, Graduate School of Biomedical Sciences, Hiroshima University, 1-2-3 Kasumi, Minami-ku, Hiroshima 734-8551, Japan

H Enzan, E Miyazaki, N Kuroda, M Toi, M Hiroi, Department of Pathology, Kochi Medical School, Kohasu, Okoh-cho, Nankoku, Kochi 783-8505, Japan

\section{REFERENCES}

1 Voung NV, Berry SC. The structure of vessels. In: Voung NV, Berry SC, eds. The pathology of vessels. Paris: Springer-Verlag France, 2002:3-23.

2 Nakayama H, Enzan H, Miyazaki E, et al. Lack of CD34 positive stromal cells within angiomyomas (vascular leiomyomas). J Clin Pathol 2002;55:395-6.

3 van de Rijn M, Rouse RV. CD34. A review. Appl Immunohistochem 1994:2:71-80.

4 Greaves MF, Brown J, Molgaard HV, et al. Molecular features of CD34: a hematopoietic progenitor cell-associated molecule. Leukemia 1992;1:31-6.

5 Simmons DL, Satterthwaite AB, Tenen DG, et al. Molecular cloning of a cDNA encoding CD34, a sialomucin of human hematopoietic stem cells. J Immunol 1992;148:267-71.

6 Lauren P. The two main types of gastric carcinoma. Diffuse and so-called intestinal type carcinomas. Acta Pathol Microbiol Scand 1965;64:31-49.

7 Nakayama H, Enzan H, Miyazaki E, et al. $\alpha$ Smooth muscle actin positive stromal cells in gastric carcinoma. J Clin Pathol 2002;55:741-4.

8 Miettinen $M$, Lindenmayer $A E$, Chaubal A. Endothelial cell markers CD31, $\mathrm{CD} 34$, and BNH9 antibody to $\mathrm{H}$ - and $\mathrm{Y}$-antigens: evaluation of their specificity and sensitivity in the diagnosis of vascular tumors and comparison with von Willebrand factor. Mod Pathol 1994;7:82-90.

9 Nakayama H, Enzan H, Miyazaki E, et al. CD34-positive stromal cells in gastric adenocarcinomas. J Clin Pathol 2001;54:846-8.

10 Sartore S, Chiavegato A, Faggin E, et al. Contribution of adventitial fibroblasts to neointima formation and vascular remodeling. Circ Res 2001;89:1111-21. 